\title{
Leg Extensor Muscle Strength, Postural Stability, and Fear of Falling After a 2-Month Home Exercise Program in Women With Severe Knee Joint Osteoarthritis
}

\author{
Monika Rätsepsoo $^{1}$, Helena Gapeyeva ${ }^{1}$, Jelena Sokk ${ }^{1}$, Jaan Ereline ${ }^{1}$, \\ Tiit Haviko ${ }^{2}$, Mati Pääsuke ${ }^{1}$ \\ ${ }^{1}$ Institute of Exercise Biology and Physiotherapy, University of Tartu, \\ ${ }^{2}$ Department of Traumatology and Orthopedics, University of Tartu, Estonia
}

Key Words: osteoarthritis; home exercise program; muscle strength; balance; falls.

Summary. Background and Objective. The aim of this study was to compare the leg extensor muscle strength, the postural stability, and the fear of falling in the women with severe knee joint osteoarthritis (OA) before and after a 2-month home exercise program (HEP).

Material and Methods. In total, 17 women aged 46-72 years with late-stage knee joint $O A$ scheduled for total knee arthroplasty participated in this study before and after the 2-month HEP with strengthening, stretching, balance, and step exercises. The isometric peak torque (PT) of the leg extensors and postural stability characteristics when standing on a firm or a foam surface for 30 seconds were recorded. The fear of falling and the pain intensity (VAS) were estimated.

Results. A significant increase in the PT and the PT-to-body weight (PT-to-BW) ratio of the involved leg as well as the bilateral PT and the PT-to-BW ratio was found after the 2-month HEP compared with the data before the HEP $(P<0.05)$. The PT and the PT-to-BW ratio of the involved leg were significantly lower compared with the uninvolved leg before the HEP $(P<0.05)$. The center of the pressure sway length (foam surface) decreased significantly after the HEP $(P<0.05)$. Significant correlations were found between the PT of the involved leg and the bilateral PT and the fear of falling and between the PT of the involved leg and the postural sway (foam surface) before the HEP.

Conclusions. After the 2-month HEP, the leg extensor muscle strength increased and the postural sway length on a foam surface decreased. The results indicate that the increased leg extensor muscle strength improves postural stability and diminishes the fear of falling in women with latestage knee joint $O A$.

\section{Introduction}

Exercise is a key component in the management of osteoarthritis (OA) symptoms and has been shown to be beneficial to patients with OA of different severity (1). A review of the most recent evidence-based studies found that strength training, aquatic therapy, and balance and perturbation therapy were most beneficial with respect to pain reduction and function improvement (2). However, many of these programs require intensive supervision and sophisticated equipment, whereas it has been found that a home exercise program for patients with knee joint OA provides important benefits (3). An easy home-based exercise program designed to improve the strength of muscles acting around the knee, range of motion at the knee joint, and the locomotor function can significantly reduce knee pain (4). Preparation for stress of total knee arthroplasty (TKA) by exercise training may improve the strength and the function before surgery and, if

Correspondence to M. Rätsepsoo, Institute of Exercise Biology and Physiotherapy, University of Tartu, Jakobi 5, 51014 Tartu, Estonia. E-mail:monika.ratsepsoo@gmail.com effective, has the potential to contribute to postoperative recovery (5). The preoperative performance of functional tasks has been shown to be a predictor of postoperative performance (6). There is also convincing evidence that exercise improves muscle sensorimotor dysfunction and reduces pain and disability without exacerbating joint damage (7).

The risk of falling increases almost 2.5 times in those with lower extremity OA compared with agematched controls. However, underlying mechanisms are not fully clear (8). Balance deficits have been associated with an increased risk of falls and poorer mobility in the elderly (9). Increased joint pain, decreased muscle strength (10), and impairments in knee joint proprioception $(10,11)$ may particularly influence postural sway. Other functional outcomes, such as the gait pattern, may also be related to falls (8). It has been previously found that muscle strength and body balance measures improve significantly following resistance training intervention (12). A short-term exercise program including resistance training using Thera-Bands, flexibility, step exercise, and light walking at least 3 times per week for $4-8$ 
weeks before TKA has been effective for increasing the strength and the function in people with severe OA. The program has also been found to be easily transferred to home environment (5).

Knee joint OA is a very common condition in the elderly. It affects women more often than men and causes functional impairment (e.g., limitations in the ability to walk, use stairs, and rise from a chair), poor quality of life, and in severe cases, leads to joint arthroplasty. Interventions aiming at muscle strengthening, postural stability training, and reduction of the fear of falling are very important not only in a short-term perspective but can possibly improve the results of operative treatment. Research on preoperative home exercise programs and their effect on leg extensor muscle strength, postural stability, and the fear of falling in women with knee joint OA has not been elucidated enough in literature.

The aim of the present study was to investigate the changes in the isometric strength of the leg extensors and the postural stability and the correlations of the isometric strength and the postural stability with the self-reported fear of falling in the women with late-stage knee joint $\mathrm{OA}$ who performed a 2-month preoperative home exercise program (HEP) before scheduled unilateral TKA.

\section{Material and Methods}

Subjects. Seventeen women aged 46-72 years with severe knee joint OA (stage 3-4 [13]) scheduled for primary TKA participated in this longitudinal study twice: before and 2 months after performing the HEP. The subjects were recruited by an orthopedic surgeon between February 2011 and August 2012. The inclusion criteria for the subjects were as follows: diagnosis of stage $3-4$ knee joint OA, scheduled unilateral TKA, independent walking without a walking aid, and motivation to perform the HEP. The exclusion criteria for the subjects included other musculoskeletal diseases, neurological diseases, and diseases affecting balance and coordination. On the day of the assessments, the subjects did not take any inflammatory or pain-relieving medications, and the assessments were conducted in the morning. All the subjects signed written consent to participate in the study voluntarily. The Ethics Committee of the University approved this study.

Home Exercise Program. The preoperative therapeutic HEP composed by a physiotherapist was oriented toward muscle strengthening exercises with an elastic band (Thera-Band, System of Progressive Exercise, USA), stretching, balance, and step exercises. The subjects received a detailed handout with pictures and descriptions of the exercises. The HEP included the same exercises for all the patients. At the first meeting, the physiotherapist taught the exercise program to the patients and gave them an elastic band set with 3 resistance levels, a printed handout, and a training diary. Once a week, the physiotherapist reached the patients by phone and asked them about the HEP and any problems with the knee (14). The patients performed the HEP for an average of 64 days (an average of 25 minutes a day) and recorded various data in their diary every day, i.e., how many times they performed each exercise, training time, perception of pain before and after the HEP, time spent in outdoor walking, and the number of stairs climbed during the day. It has been previously shown that the implementation of a resistance band and a step exercise program can increase the leg strength in the surgical leg, decrease the leg strength asymmetry (5), improve balance (12), and increase the ability to perform functional tasks for individuals with severe OA before TKA (5). Balance therapy has been found to be beneficial with respect to pain reduction and function improvement (9).

Postural Sway Measurement. For the assessment of the postural stability, the subjects stood bipedally on a force plate (Kistler 9286A, Switzerland) on a firm surface and a foam surface (balance pad) for 30 seconds. The following postural sway characteristics were recorded using the SWAY ${ }^{\circledR}$ software of the movement analysis system Elite Clinic (BTS S.p.A., Italy): the center of the pressure (COP) sway in the anterior-posterior (AP) and the mediolateral (ML) sway $(\mathrm{mm})$ direction; the sway length $(\mathrm{mm})$, the radius $(\mathrm{mm})$, and the area $\left(\mathrm{mm}^{2}\right)$. All the characteristics were adjusted to the subject's height $(\mathrm{m})$ and foot length ( $\mathrm{mm})(15)$.

Leg Extensor Muscle Strength Measurement. For the measurement of the isometric strength of the leg extensors, the subjects were seated into a specially designed dynamometric chair in a sitting posture with the angles of knee and hip flexion maintained at $110^{\circ}$ to $120^{\circ}$, respectively (16). The closed-chain assessment of the entire lower-limb strength rather than the open-chain measures of the knee extensor strength may provide greater insight into functional limitations after TKA (17).

The body position of the subjects was secured by a Velcro belt placed over the hip to avoid sliding forward. The feet were placed on a footplate (connected to a strain gauge) mounted on a steel bar held in ball bearings on the frame. The isometric strength of the leg extensor muscles was recorded by a standard strain gauge transducer (1778 DST2, Russia). During testing, the subjects pushed the footplate with maximal effort. Three trials were measured (bilaterally and unilaterally) with a rest period of 1 minute, and the best result was taken into consideration. The peak torque (PT) in $\mathrm{N} \cdot \mathrm{m}$ and the peak torque-to-body weight (PT-to-BW) ratio in $\mathrm{N} \cdot \mathrm{m} \cdot \mathrm{kg}^{-1}$ were calculated. All the measure- 
ments were initially performed on the less involved leg (uninvolved leg) and then on the leg scheduled for surgery (involved leg).

Fear of Falling Assessment. The Modified Falls Efficacy Scale (MFES) questionnaire was used to estimate the fear of falling. The scale was explained to the subjects. The items on the scale are scored from 0 to 10 points, with 0 points meaning "not confident/not sure at all," 5 points meaning "fairly confident/fairly sure," and 10 points meaning "completely confident/completely sure." The subjects were asked the question, "How confident/sure are you that you can do each of the activities without falls?" (18). The score was calculated as an average score divided by the number of the items rated (19). The MFES has been shown to have a high retest reliability in older people who experience falls and those who not (18).

Knee Joint Pain Assessment. The self-reported knee joint pain intensity in both knees was assessed by a visual analogue scale (VAS), where 0 points indicates no pain and 10 points indicates maximal pain (VAS score values in $\mathrm{mm}$ were transformed to the point scale where $10 \mathrm{~mm}$ was equal to 1 point). The general knee joint pain intensity was assessed before and after the 2-month HEP. The patients assessed the knee joint pain intensity directly before, during, and after exercising, but the data are not discussed in the present article. For patients who underwent TKA, the standard VAS version (0- to 10 -point scale) is more reliable than other knee pain questionnaires (20).

Statistical Analysis. The data are presented by means and standard errors (SE). One-way analysis of variance (ANOVA) followed by Turkey post hoc comparisons was used to evaluate the differences between the involved and the uninvolved leg. A paired $t$ test was used to evaluate the differences between pre- and post-HEP sessions. The Pearson correlation coefficients were calculated to examine correlation between the quantitative variables. The relationship between the leg extensor muscle strength, the postural sway, and the fear of falling characteristics was found by the Spearman rank correlation or Spearman $\varrho$. $P$ values of $<0.05$ were regarded as statistically significant.

\section{Results}

Leg Extensor Muscle Strength. The PT of the involved leg was significantly lower compared with the uninvolved leg before the HEP $(P<0.01)$, but after the 2-month HEP, there were no significant differences between the legs. After the 2-month HEP, the PT of the involved leg increased significantly compared with that before the HEP $(P<0.05)$ (Fig. 1A). After the 2-month HEP, the bilateral PT increased significantly compared with that before the HEP (from $533.8 \pm 62.7$ to $679.3 \pm 101.7 \mathrm{~N} \cdot \mathrm{m}, P<0.05$ ).

The PT-to-BW ratio of the involved leg was significantly lower compared with the uninvolved leg before the HEP $(P<0.01)$, but no significant difference was found between the legs after the 2-month
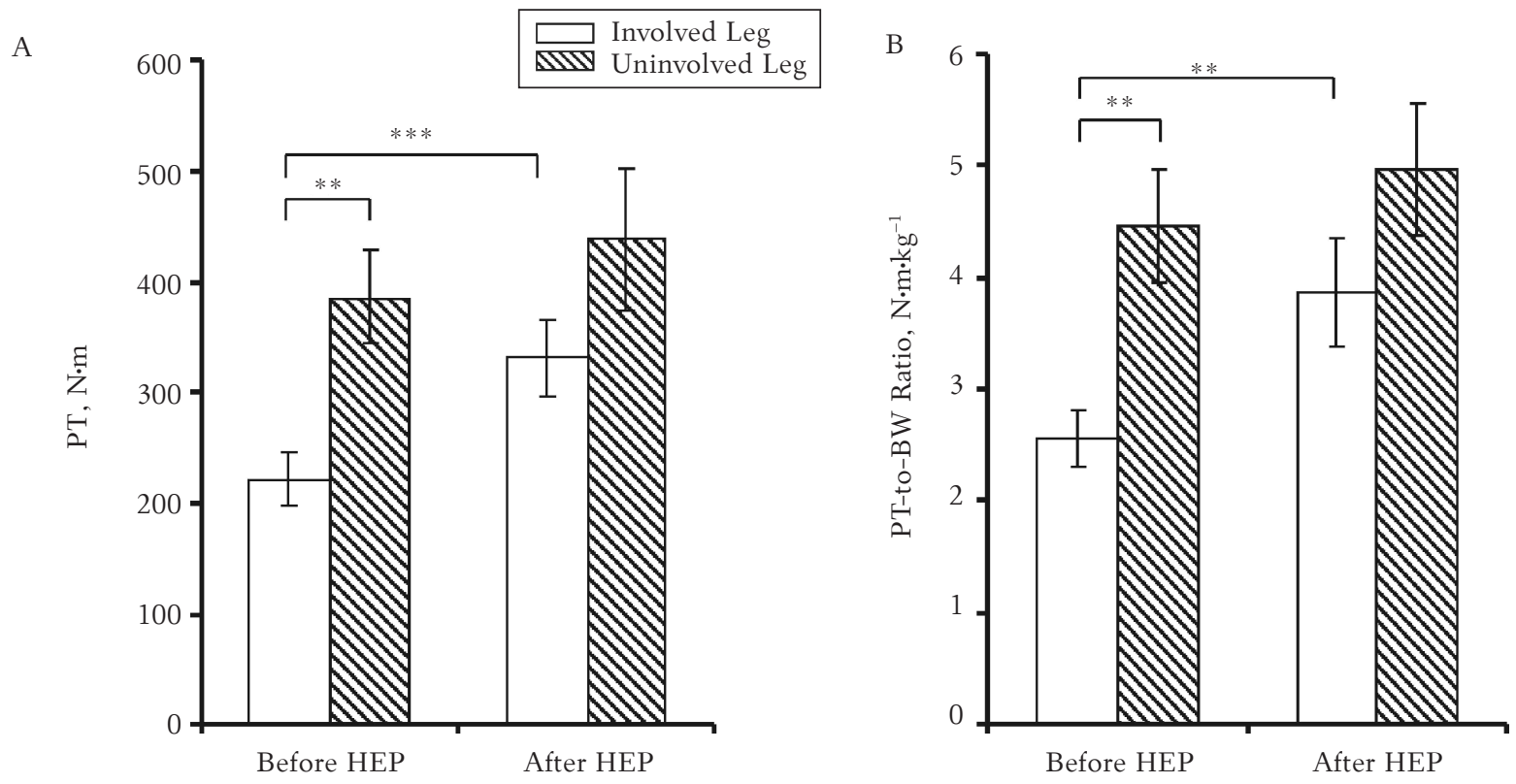

Fig. 1. The isometric peak torque (PT) (A) and the peak torque-to-body weight (PT-to-BW) ratio (B) of the leg extensor muscles of the involved and uninvolved legs in the women with knee joint osteoarthritis before and after the 2-month home exercise program (HEP)

Values are means and error bars indicate standard error. $* * P<0.01$, *** $P<0.001$. 
HEP. The PT-to-BW ratio of the involved leg increased significantly after the HEP compared with that before the HEP $(P<0.05)$ (Fig. 1B). The bilateral PT-to-BW ratio increased significantly after the 2-month HEP compared with that before the HEP (from $6.1 \pm 0.7$ to $7.7 \pm 0.9 \mathrm{~N} \cdot \mathrm{m}^{\cdot \mathrm{kg}^{-1}}, P<0.05$ ).

Postural Stability. The women with knee joint OA demonstrated a significant decrease in the sway length when standing on a foam surface after the 2-month HEP $(P<0.05)$ (Fig. 2D). The COP sway in the AP and $\mathrm{ML}$ directions and the sway radius when standing on a firm or a foam surface and the sway length on a firm surface did not differ significantly after the HEP (Fig. 2A-C). In the COP sway area, no significant changes were found after the HEP (from 0.16 \pm 0.03 to $0.14 \pm 0.02 \mathrm{~mm}^{-1}$ on a firm surface and from $0.61 \pm 0.09$ to $0.53 \pm 0.07 \mathrm{~mm}^{-1}$ on a foam surface).
Fear of Falling and Knee Joint Pain. The MFES score did not differ significantly after the 2-month HEP compared with that before the HEP $(8.2 \pm 0.6$ and $7.9 \pm 0.6$ points, respectively; $P>0.05)$. The knee joint pain intensity of the involved leg according to the VAS did not differ significantly after the 2-month HEP compared with that before the HEP (4.4 \pm 0.5 and $5.0 \pm 0.4$ points, respectively; $P>0.05$ ).

Correlations. A significant negative correlation was noted between the COP sway radius and the PT of the involved leg $(r=-0.50, P<0.05)$, but the correlation between the sway area and the PT of the involved leg before the HEP $(r=-0.40, P>0.05)$ was not significant (Fig. 3).

Correlations Between Fear of Falling and Other Measured Characteristics. Before the HEP, significant correlations were found between the PT of the
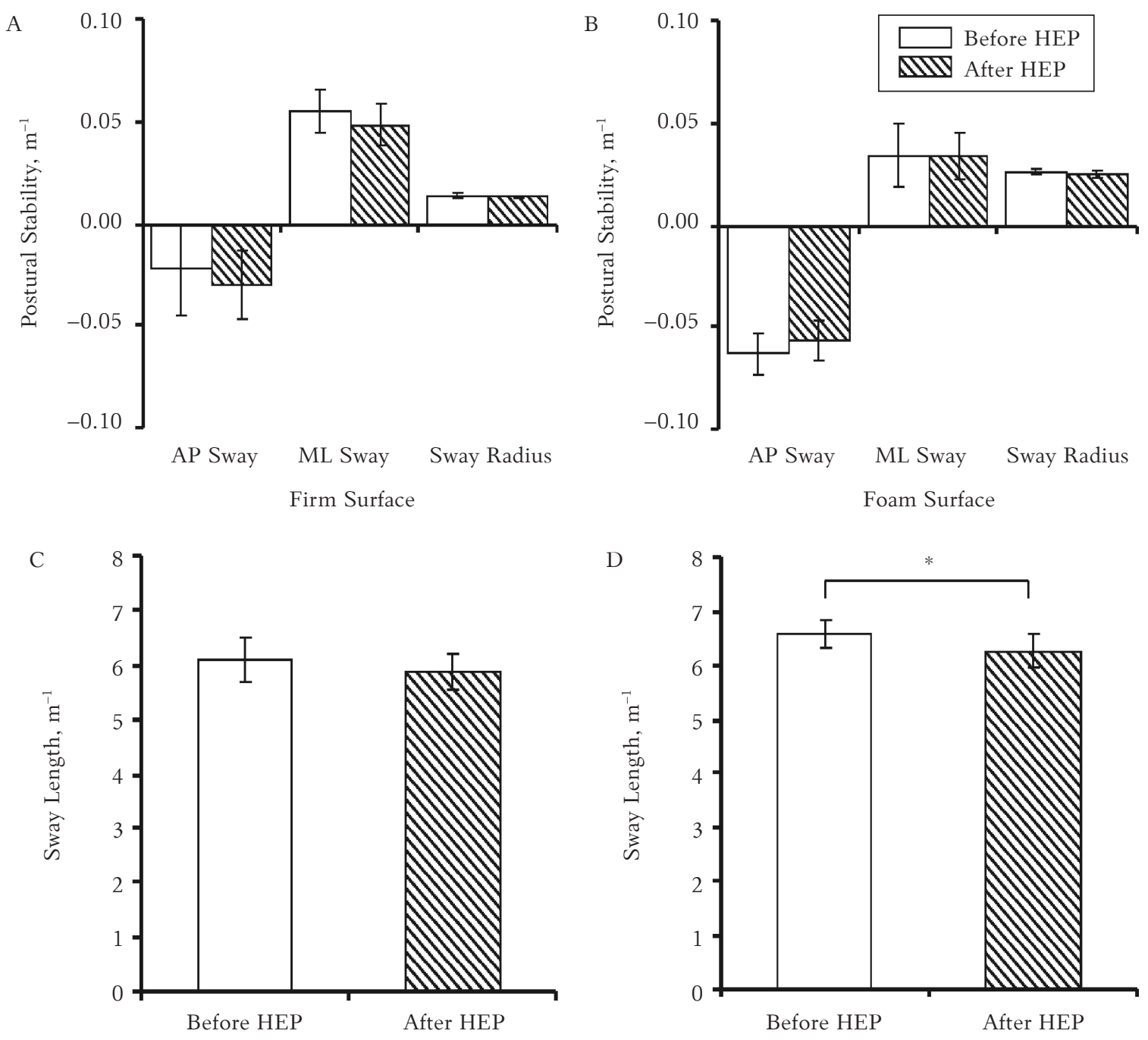

Firm Surface

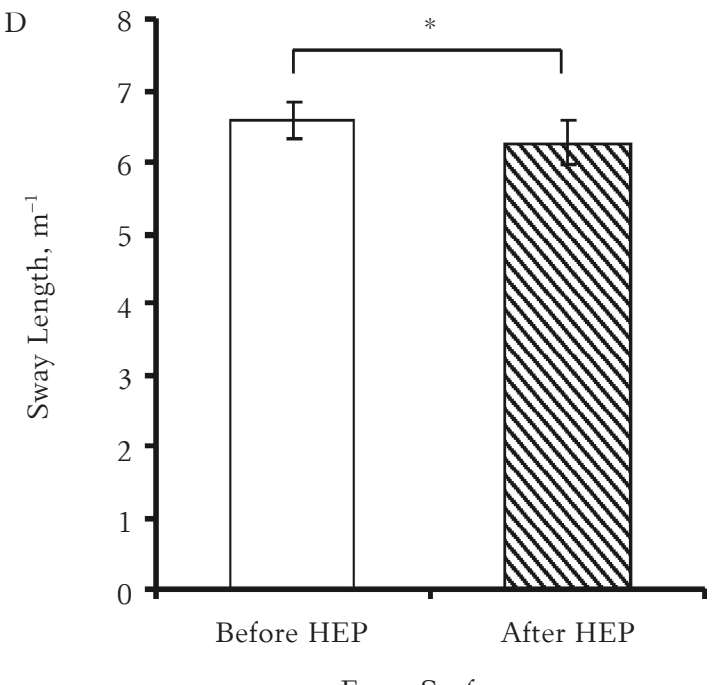

Fig. 2. The center of the pressure (COP) sway in the anterior-posterior (AP) and mediolateral (ML) directions, the sway radius, and the length of quiet standing on a firm and a foam surface in the women with knee joint osteoarthritis before and after the 2-month home exercise program (HEP)

Values are means and error bars indicate standard error. $* P<0.05$. 

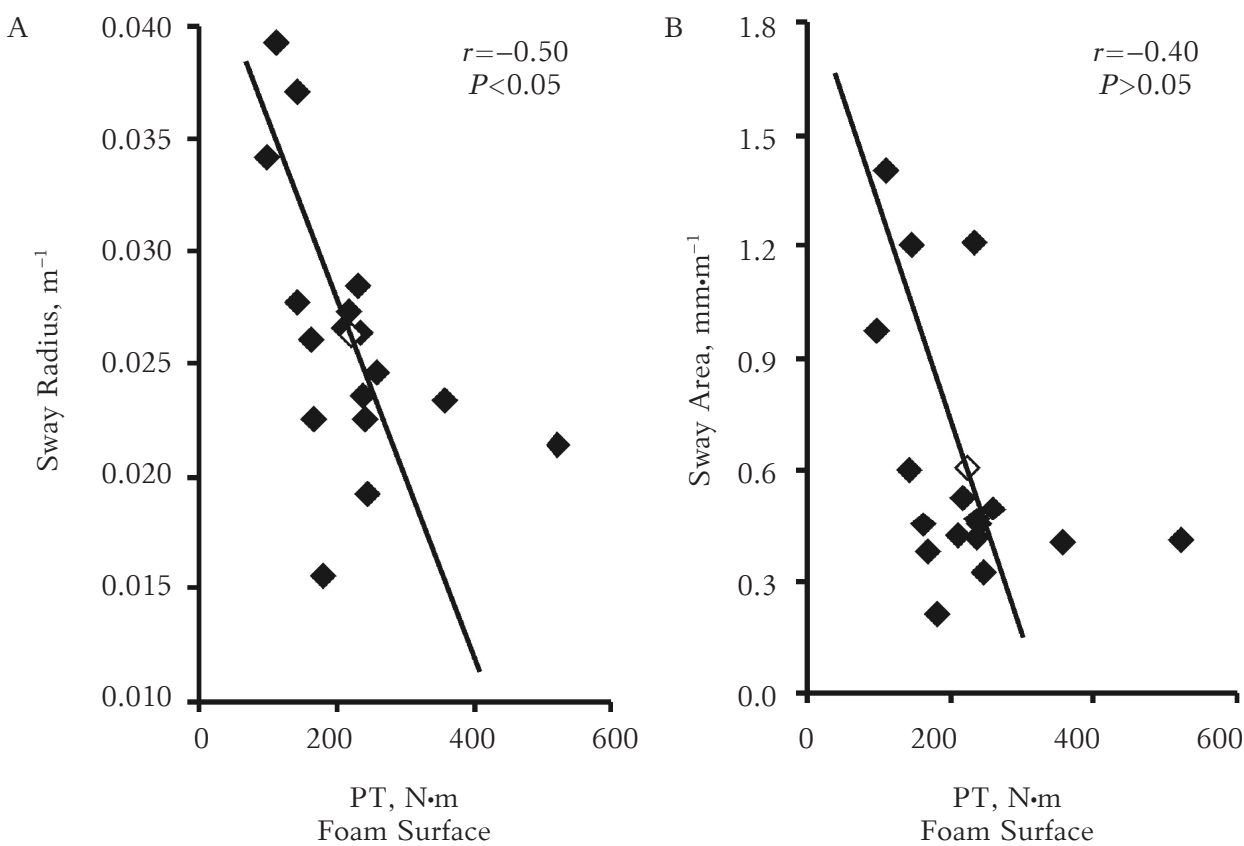

Fig. 3. The correlations between the isometric peak torque (PT) of the leg extensor muscles of the involved leg and the center of the pressure sway radius (A) and the area (B) when standing on a foam surface in the women with knee joint osteoarthritis (OA) before the 2-month home exercise program

Table. Correlations Between Leg Extensor Muscle Strength, Postural Stability, Knee Joint Pain Intensity, and Fear of Falling in Women With Late-Stage Knee Joint Osteoarthritis $(\mathrm{N}=17)$

\begin{tabular}{|c|c|c|}
\hline & $\begin{array}{l}\text { VAS } \\
\text { Score }\end{array}$ & $\begin{array}{l}\text { MFES } \\
\text { Score }\end{array}$ \\
\hline PT of involved leg, N·m & $-0.54^{*}$ & $0.46^{*}$ \\
\hline PT-to-BW ratio of involved leg, $\mathrm{N} \cdot \mathrm{m} \cdot \mathrm{kg}^{-1}$ & -0.29 & $0.67^{*}$ \\
\hline Bilateral PT, N·m & 0.01 & $0.50 *$ \\
\hline Sway length, $\mathrm{m}^{-1}$ & $0.52 *$ & -0.37 \\
\hline ML sway, $\mathrm{m}^{-1}$ & $0.51 *$ & 0.04 \\
\hline
\end{tabular}

PT, peak torque; PT-to-BW ratio, peak torque-to-body weight ratio; ML, mediolateral; VAS, visual analogue scale;

MFES, Modified Falls Efficacy Scale.

$* P<0.05$.

involved leg and the VAS score $(\varrho=-0.54, P<0.05)$ and the MFES score $(\varrho=0.46, P<0.05)$. Moreover, a significant correlation was noted between the MFES score and the PT-to-BW ratio of the involved leg $(\varrho=0.67, P<0.05)$ as well as the bilateral PT $(\varrho=0.50, P<0.05)$ (Table). The VAS score of pain intensity had a significant association with the COP sway radius on a foam surface before the HEP $(\varrho=0.52, P<0.05)$. After the 2 -month HEP, a significant correlation was found between the ML sway and the VAS score $(\varrho=0.51, P<0.05)$ (Table).

\section{Discussion}

In the present study, the changes in the leg extensor muscle strength, the postural stability, and the fear of falling as well as the relationship between these characteristics were analyzed before and after the 2-month HEP in the women with severe knee joint OA scheduled for TKA. The main findings of this study were the following: 1 ) after the 2-month HEP, the isometric strength of the leg extensor muscles increased compared with that before the HEP; 2) the COP sway length when standing on a foam surface decreased, whereas no significant changes in other postural stability characteristics measured when standing on a firm or a foam surface after the 2-month HEP were noted; and 3) a significant correlation between the unilateral and the bilateral leg extensor muscle strength and the fear of falling was found.

In the present study, the complex 2-month HEP was used with a focus not only on strengthening exercises with additional resistance, but also on stretching, balance, and step exercises under weekly supervision of the professional physiotherapist. A complex assessment of the patients' status was performed before and after the 2-month HEP. The muscle strength was investigated in the whole lower limb extensor muscles as compared with classical studies of the knee extensor muscles. The novelty of the study gives an estimation of postural sway characteristics on 2 different surfaces (firm and foam). The influence of anthropometric characteristics was eliminated, i.e., the postural sway characteristics were adjusted to the subjects' height $(\mathrm{m})$ and foot length $(\mathrm{mm})$ and the muscle strength to the subjects' body weight $(\mathrm{kg})$. The main limitation of the 
present study was a small sample size that may limit the strength of the conclusions of this study. Another possible limitation was the duration of preoperative follow-up. With a longer follow-up and the continued HEP, the patients might have continued to recover in the measured characteristics.

In the present study, the isometric leg extensor muscle strength increased significantly after the 2-month HEP in the women with knee joint OA scheduled for primary unilateral TKA. The unilateral PT of the involved leg and the bilateral PT increased remarkably compared with those before the HEP (by 50\% and 27.3\%, respectively). Analogous changes were noted in the PT-to-BW ratio of the involved leg and the bilateral PT-to-BW ratio (a significant increase by $52 \%$ and $25.9 \%$, respectively). It has been previously found that people with knee OA compared with age- and sex-matched controls exert less force during voluntary isometric contraction of the quadriceps muscle, have less percentage activation of the quadriceps muscle, and also differ in the ratio of quadriceps strength to weight (10). Isokinetic and isometric PT loss of the knee flexors and extensors has been found in people with knee OA compared with controls (21). Strength measures improve significantly following resistance and strength training intervention $(12,22)$, and home-based strength training can produce substantial improvements in strength, pain levels, physical function, and quality of life in patients with knee OA (23). In a previous study, individuals with knee OA who undertook a program involving strength training for 8 weeks demonstrated significant improvements in the knee muscle torque (22). In the present study, the PT of the involved leg was significantly lower $(42.6 \%)$ compared with the uninvolved leg before the HEP. After the 2-month HEP, the data did not differ significantly between the legs (a difference of $24.4 \%$ ). The PT-to-BW ratio of the involved leg was significantly lower (42.7\%) compared with the uninvolved leg before the HEP but did not differ between the legs after the HEP (a difference of $21.7 \%$ ). A previously studied prehabilitation exercise program had the greatest impact on the surgical leg with a little, if any, measurable impact on the nonsurgical leg. The extension and the flexion strength of the surgical leg before the exercise program was weaker in comparison with the nonsurgical leg. This asymmetry in the strength between the surgical and the nonsurgical leg was reduced after the exercise program (5). The results of that study (5) and the present study indicate that leg strength asymmetry may be reduced through the introduction of preoperative exercises before TKA.

In the present study, a significant decrease in the COP sway length was noted when standing on a foam surface after the 2-month HEP compared with that before the HEP. It has been previously found that therapeutic exercises improve balance in women with knee OA (24). Subjects with knee OA compared with controls sway more in both the lateral and anteroposterior directions (10). Moreover, an increase in the length and area of postural sway as well as postural sway velocity has been reported in people with knee OA as compared with healthy subjects. It has been proposed that patients with OA should be referred to participate in balance exercise (11). In the present study, a significant correlation was found between the unilateral PT of the involved leg and the postural stability characteristics when standing on a foam surface (sway radius). Quadriceps strength (the ratio of maximal voluntary contraction to body weight) and proprioception are clearly important for balance control, and both are compromised in people with knee OA (10). An increase in postural sway may be due to an impairment in both or one of these parameters. Therefore, muscle weakness resulting from the OA process, muscle dysfunction secondary to pain, and the additional effect of an increased weight might all contribute to reduction in the ability of the muscle to maintain postural stability and, thus, lead to an observed increase in the frequency of sway (10). Strength and balance measures improve significantly following resistance training intervention, whereas it has been observed that there is a significant association between balance and strength outcomes (12). In the present study, there also was a significant correlation between the postural stability (the sway radius and the COP sway in the ML direction on a foam surface) and the VAS score of the pain intensity, which may explain why there were no significant changes in most postural stability characteristics as the knee joint pain did not decrease after the 2-month HEP. Previous studies have also found that knee joint pain intensity is related to postural stability (10).

In the present study, no significant difference in the self-reported fear of falling (the MFES score) was noted between the 2 measurements, i.e., there was a $4.2 \%$ increase after the 2-month HEP compared with that before the HEP. Meanwhile, taskspecific training has been found to reduce falls efficiently in middle-aged and older women with OA of lower extremities (8). Previous studies have shown that multiple functional outcomes, e.g., gait and balance impairments and muscle weakness, are related to falls (8). In the present study, a significant positive correlation was found between the leg extensor muscle strength and the MFES score. Therefore, an increase in the leg extensor muscle strength should have reduced the fear of falling in the women with knee joint OA. A more intensive preoperative approach should probably be recommended to reduce the fear of falling (and improve the MFES score) in persons with knee joint OA before TKA. 


\section{Conclusions}

The 2-month home exercise program led to increased leg extensor muscle strength in the women with severe knee joint osteoarthritis scheduled for primary unilateral total knee arthroplasty. The postural stability when standing on a firm surface was not influenced by the home exercise program, whereas the postural stability when standing on a foam surface was improved by the home exercise program. The home exercise program did not influence the self-estimated fear of falling. The results of the study confirmed that the postural stability and the self-estimated fear of falling were influenced by the leg extensor muscle strength in the women with late-stage knee joint osteoarthritis. A more intensive preoperative approach should be recommended to improve the abovementioned

\section{References}

1. Bennell KL, Hinman RS. A review of the clinical evidence for exercise in osteoarthritis of the hip and knee. J Sci Med Sport 2011;14:4-9.

2. Brakke R, Singh J, Sullivan W. Physical therapy in persons with osteoarthritis. PM R 2012;4:S53-8.

3. Deyle GD, Allison SC, Matekel RL, Ryder MG, Stang JM, Gohdes DD, et al. Physical therapy treatment effectiveness for osteoarthritis of the knee: a randomized comparison of supervised clinical exercise and manual therapy procedures versus a home exercise program. Phys Ther 2005;85: 1301-17.

4. Thomas KS, Muir KR, Doherty M, Jones AC, O’Reilly SC, Bassey EJ. Home based exercise programme for knee pain and knee osteoarthritis: randomised controlled trial. BMJ 2002;325:752.

5. Swank AM, Kachelman JB, Bibeau W, Quesada PM, Nyland J, Malkani A, et al. Prehabilitation before total knee arthroplasty increases strength and function in older adults with severe osteoarthritis. J Strength Cond Res 2011;25:318-25.

6. Mizner RL, Petterson SC, Stevens JE, Axe MJ, SnyderMackler L. Preoperative quadriceps strength predicts functional ability one year after total knee arthroplasty. J Rheumatol 2005;32:1533-9.

7. Hurley MV. Muscle dysfunction and effective rehabilitation of knee osteoarthritis: what we know and what we need to find out. Arthritis Rheum 2003;49:444-52.

8. Mackenzie HL, Rosenblatt NJ, Hurt CP, Crenshaw J, Grabiner MD. Does lower extremity osteoarthritis exacerbate risk factors for falls in older adults? Womens Health (Lond Engl) 2012;8:685-98.

9. Shumway-Cook A, Baldwin M, Polissar NL, Gruber W. Predicting the probability for falls in community-dwelling older adults. Phys Ther 1997;77:812-9.

10. Hassan BS, Mockett S, Doherty M. Static postural sway, proprioception and maximal voluntary quadriceps contraction in patients with knee osteoarthritis and normal control subjects. Ann Rheum Dis 2001;60:612-8.

11. Tarigan TJE, Kasjmir YI, Atmakusuma D, Lydia A, Bashiruddin J, Kusumawijaya K, et al. The degree of radiographic abnormalities and postural instability in patients with knee osteoarthritis. Acta Med Indones 2009;41:15-9.

12. Orr R. Contribution of muscle weakness to postural stability in the elderly. A systematic review. Eur J Phys Rehabil Med 2010;46:183-220. functions in people with knee joint osteoarthritis before total knee arthroplasty. In future studies, it is necessary to analyze the effect of a preoperative home exercise program on postoperative recovery after total knee arthroplasty in women with knee osteoarthritis.

\section{Acknowledgments}

This study was partly supported by the Estonian Ministry of Education and Research project SF0180030s07, the Estonian Science Foundation project 7939, and the European Union FP7 223576 project. We thank Ms. Tatjana Kums for assistance in data collection.

\section{Statement of Conflict of Interest}

The authors state no conflict of interest.

13. Kellgren JH, Lawrence JS. Radiological assessment of osteo-arthrosis. Ann Rheum Dis 1957;16:494-501.

14. Mazieres B, Thevenon A, Coudeyre E, Chevalier X, Revel M, Rannou F. Adherence to, and results of, physical therapy programs in patients with hip or knee osteoarthritis. Development of French clinical practice guidelines. Joint Bone Spine 2008;75:589-96.

15. Chiari L, Rocchi L, Cappello A. Stabilometric parameters are affected by anthropometry and foot placement. Clin Biomech (Bristol, Avon) 2002;17:666-7.

16. Raudsepp L, Pääsuke M. Gender differences in fundamental movement patterns, motor performance, and strength measurements of prepubertal children. Ped Exerc Sci 1995;7:294-304.

17. Rossi MD, Hasson S, Kohia M, Pineda E, Bryan W. Relationship of closed and open chain measures of strength with perceived physical function and mobility following unilateral total knee replacement. J Geriatr Phys Ther 2007;30: 23-7.

18. Hill KD, Schwarz JA, Kalogeropoulos AJ, Gibson SJ. Fear of falling revisited. Arch Phys Med Rehabil 1996;77:1025-9.

19. Hill K, Schwarz J, Flicker L, Carroll S. Falls among healthy community dwelling older women: a prospective study of frequency, circumstances, consequences and prediction accuracy. Aust N Z J Public Health 1999;23:41-8.

20. Boeckstyns ME, Backer M. Reliability and validity of the evaluation of pain in patients with total knee replacement. Pain 1989;38:29-33.

21. Tan J, Balci N, Sepici V, Gener FA. Isokinetic and isometric strength in osteoarthrosis of the knee. Am J Phys Med Rehabil 1995;74:364-9.

22. Jan MH, Lin JJ, Liau JJ, Lin JF, Lin DH. Investigation of clinical effects of high- and low-resistance training for patients with knee osteoarthritis: a randomized controlled trial. Phys Ther 2008;88:427-36.

23. Baker KR, Nelson ME, Felson DT, Layne JE, Sarno R, Roubenoff R. The efficancy of home based progressive strength training in older adults with knee osteoarthritis: a randomized controlled trial. I Rheumatol 2001;28:1655-65.

24. Silva A, Serrão PRMS, Driusso P, Mattiello S M. The effects of therapeutic exercise on the balance of women with knee osteoarthritis: a systematic review. Rev Bras Fisioter 2012;16:1-9. 